PROFESIONALES Y HERRAMIENTAS PARA EL DESARROLLO LOCAL Y SUS SINERGIAS TERRITORIALES. EVALUACIÓN Y PROPUESTAS DE FUTURO IX Coloquio Nacional de Desarrollo Local del GTDL-AGE 

ANTONIO MARTÍNEZ PUCHE, XAVIER AMAT MONTESINOS, ISABEL SANCHO CARBONELL y DANIEL SANCHIZ CASTAÑO (EDS.)

\section{PROFESIONALES Y HERRAMIENTAS PARA EL DESARROLLO LOCAL Y SUS SINERGIAS TERRITORIALES. EVALUACIÓN Y PROPUESTAS DE FUTURO}

IX Coloquio Nacional de Desarrollo Local del GTDL-AGE

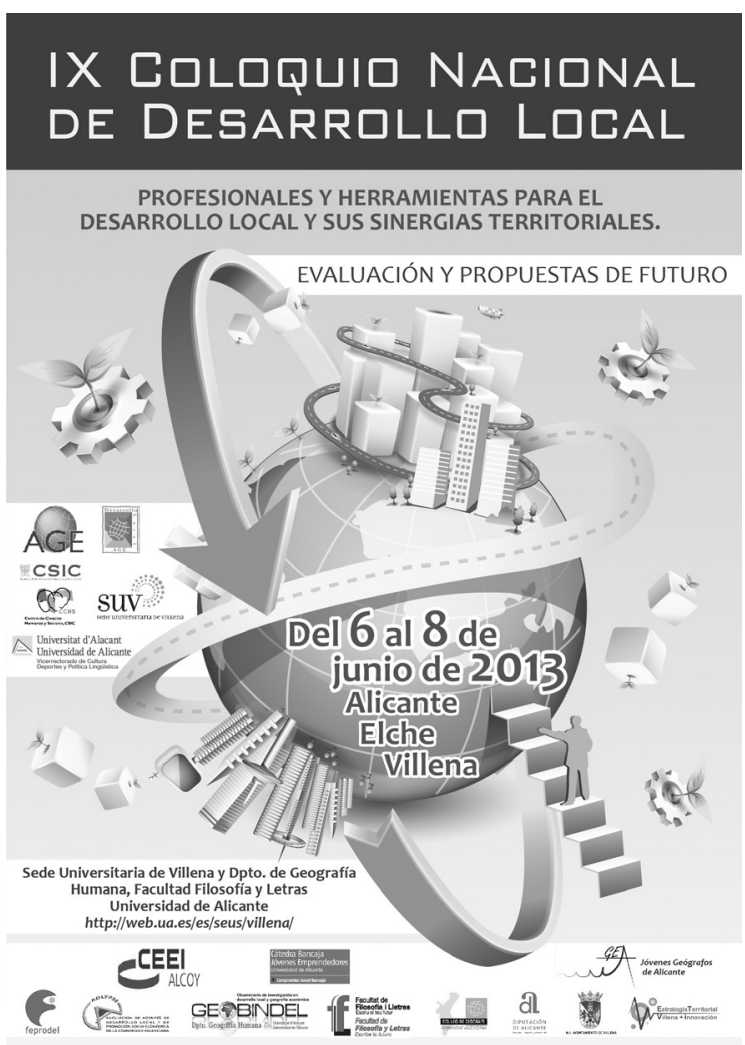


Este libro ha sido examinado y valorado por evaluadores ajenos a la Universidad de Alicante, con el fin de garantizar la calidad científica del mismo.

Publicacions de la Universitat d'Alacant

03690 Sant Vicent del Raspeig

Publicaciones@ua.es

http://publicaciones.ua.es

Telèfon: 965903480

(C) Antonio Martínez Puche, Xavier Amat Montesinos,

Isabel Sancho Carbonell y Daniel Sanchiz Castaño (eds.), 2016

(C) d'aquesta edició: Universitat d'Alacant

ISBN: 978-84-16724-00-0

Dipòsit legal: A 92-2016

Disseny de coberta: candela ink

Composició: Página Maestra (Miguel Ángel Sánchez Hernández)

Impressió i enquadernació: Guada Impresores

\section{unte \\ Unión de Editoriales
Universitarias Españolas \\ WWW.une.es
WWA}

Esta editorial es miembro de la UNE, cosa que garantiza la difusión y comercialización nacional y internacional de sus publicaciones.

Reservados todos los derechos. Cualquier forma de reproducción, distribución, comunicación pública o transformación de esta obra sólo puede ser realizada con la autorización de sus titulares, salvo excepción prevista por la ley. Diríjase a CEDRO (Centro Español de Derechos Repográficos, www.cedro.org) si necesita fotocopias o escanear algún fragmento de esta obra. 


\title{
DESARROLLO RURAL ENDÓGENO, COMUNIDAD, PARTICIPACIÓN E IDENTIDAD: LUCES Y SOMBRAS EN EL MARCO DE LA INICIATIVA LEADER ${ }^{1}$
}

\author{
José María Martínez Navarro \\ Facultad de Ciencias de la Educación y Humanidades. \\ Universidad de Castilla-La Mancha \\ josemaria.martinez@uclm.es \\ Carmen Vázquez Varela \\ Facultad de Ciencias de la Educación y Humanidades. \\ Universidad de Castilla-La Mancha \\ carmen.vazquez@uclm.es
}

\section{RESUMEN}

La intención de la iniciativa LEADER ha sido poner en marcha la capacidad de construcción de los actores locales mediante la activación del capital social. Los agentes locales del programa deberían desarrollar una actitud cooperativa, un alto nivel de confianza mutua, y una determinación compartida para trabajar sobre objetivos comunes. Sin embargo, la mayor parte de los grupos LEADER se han propuesto como objetivo la creación de empleo así como de resultados económicos cuantificables y sólo una minoría se ha centrado en objetivos "procesuales", todo ello en el marco de escalas temporales demasiado limitadas.

Palabras clave: iniciativa LEADER, participación, identidad, Manchuela conquense, Serranía de Cuenca.

1 Esta comunicación es parte del programa de difusión de los resultados del proyecto de investigación Banco territorial de información sobre capital social. Sistema geoespacial de análisis dinámico sobre el ámbito rural de la provincia de Cuenca, financiado por la Consejería de Educación y Ciencia. Junta de Comunidades de Castilla-La Mancha, con el número de referencia: PAI-05-057. 


\title{
ENDOGENOUS RURAL DEVELOPMENT, COMMUNITY, PARTICIPATION AND IDENTITY: LIGHTS AND SHADOWS IN THE FRAMEWORK OF THE LEADER INITIATIVE
}

\begin{abstract}
The intention of the LEADER Initiative has been to activate the capacitybuilding of local actors through the activation of social capital, therefore local interpreters of the programme should develop a cooperative attitude, a high level of mutual trust, and a shared determination to work on common objectives. However, in practice most of the LEADER groups in Europe have pursued the creation of employment and quantifiable economic results while only a minority has focused on "processual" objectives. The time scales have been too limited, the monitoring and advising criteria have been financial and tangible results-oriented.
\end{abstract}

Key words: LEADER Initiative; participation; identity; Manchuela conquense, Serranía de Cuenca.

\section{A MODO DE INTRODUCCIÓN}

El desarrollo endógeno en tanto que proceso que contribuye a construir la fortaleza de los territorios frente a las grandes tendencias de la competencia global, la crisis fiscal o la exclusión social ha sido objeto de constante discusión en la producción académica reciente. Por otra parte, la utilización del concepto en el marco de la iniciativa LEADER se ha vinculado a la capacidad de respuesta del conjunto de la población entendido como un agente diferenciado de cada uno de los individuos que la integran, para lograr este objetivo se ha tratado de mejorar las habilidades de la comunidad, animar nuevas formas de organización, estimular nuevas vías de vinculación entre grupos e instituciones públicas y ayudar tanto a los individuos como a las organizaciones a lograr mayor flexibilidad y capacidad de adaptación a situaciones cambiantes. En la práctica esto ha significado apoyar el desarrollo de las comunidades rurales -entendido como una aproximación al trabajo con y al desarrollo de las capacidades de los individuos y grupos dentro de sus propias comunidades. Esta perspectiva apuesta por reforzar a las comunidades rurales mediante el apoyo a la adquisición de confianza de su propia población, así como del conocimiento y las habilidades de las que disponen, sin olvidar la capacidad para trabajar juntos. En otras palabras, la esencia de programa LEADER podría caracterizarse, en parte, como la creación de varias formas de capital social que podrían beneficiar al conjunto de la comunidad.

Siguiendo esta argumentación, y en palabras de COHEN (1985), la noción de comunidad ha disfrutado de un amplio significado retórico, un hecho que 
tiene implicaciones positivas pero también sus riesgos. Los técnicos que desarrollan su trabajo en beneficio del desarrollo rural podrían haber tratado de animar y desarrollar su construcción simbólica, de hecho, RAY (1999) afirma que su construcción simbólica es la esencia de la iniciativa LEADER, no obstante, es vital reconocer y gestionar los intereses en conflicto que existen dentro de esas "comunidades" simbólicamente construidas o "territorios culturales", pues su ocultación puede contribuir a la exclusión (SHUCKSMITH, 2000).

Se supone que, desde el punto de vista de la Comisión Europea, la intención de la iniciativa LEADER ha sido poner en marcha la capacidad de construcción de los actores locales mediante la activación del capital social, por lo tanto, los agentes locales del programa deberían desarrollar una actitud cooperativa, un alto nivel de confianza mutua, y una determinación compartida para trabajar sobre objetivos comunes. Sin embargo, en la práctica la mayor parte de los grupos LEADER en Europa se han propuesto como objetivo la creación de empleo así como de resultados económicos cuantificables mientras sólo una minoría se ha centrado en objetivos "procesuales". La verdad es que, aunque el capital social "colectivo" podría ser considerado el objetivo marco de la iniciativa LEADER, las instituciones nacionales, regionales y europeas no lo han favorecido con pasos específicos. Las escalas temporales han sido demasiado limitadas, el control y los criterios de asesoramiento han estado orientados por aspectos financieros y orientados a los resultados antes que preocupados por el proceso de construcción del capital social (ÁRNASON et al., 2009).

Por otro lado, el capital social está muy vinculado a los conceptos de identidad y lugar. Allí donde el capital social aporta beneficios positivos, tiene muchas posibilidades de estar asociado con una pluralidad de identidades culturales, una mezcla y entramado de escalas espaciales, y fuertes lazos con los múltiples temas históricos que caracterizan a las zonas rurales europeas. (ÁRNASON et al., 2009).

Trataremos de verificar buena parte de estas hipótesis utilizando como laboratorio dos territorios vinculados a Grupos de Acción Local que han desarrollado distintos programas LEADER en la provincia de Cuenca, recurriendo para ello a una mezcla de metodologías mixtas cuantitativo-cualitativas. Todo ello se inscribe en el marco del proyecto de investigación Banco territorial de información sobre capital social. Sistema geoespacial de análisis dinámico sobre el ámbito rural de la provincia de Cuenca, posteriormente ampliado al conjunto de las cinco provincias de la región, que ante la carencia de estudios empíricos sobre la existencia y características del capital social en Castilla-La Mancha, ha planteado la recogida, elaboración y análisis de datos cuantitativos y cualitativos georeferenciados a escala municipal, mediante la recogida de la información cuantitativa disponible, la realización de encuestas y entrevistas en profundidad y las sesiones de trabajo con grupos de expertos. 


\section{FuENTES Y METODOLOGÍA}

Esta comunicación aborda el análisis de parte de los resultados de un proyecto de investigación que ha tratado de identificar y analizar las relaciones sociales que enmarcan las actividades productivas con el objetivo de entender el funcionamiento de las iniciativas comunitarias así como la reconfiguración de los sistemas socio-productivos en un nuevo contexto económico. Para el propósito de esta aportación, hemos seleccionado la primera de las cinco provincias analizadas, Cuenca, que se extiende sobre una superficie de $17.141 \mathrm{~km}^{2}$ con una densidad de población en 2012 de $12,7 \mathrm{~h} / \mathrm{km}^{2}$ (la población para este mismo año alcanzó los 218.036 habitantes). Aunque la provincia se articula en cinco Grupos de Acción Local, que reúnen 238 municipios, nuestro análisis comparado se centra en los dos únicos vinculados en sentido estricto a la iniciativa comunitaria LEADER (ver mapa 1), el primero de ellos PRODESE (Promoción y Desarrollo Serrano), que desde 1991 comienza a gestionar el LEADER 1 siendo beneficiario de las dos siguientes convocatorias (LEADER 2 y LEADER +), y el segundo ADIMAN (Asociación de desarrollo Integral de la Manchuela conquense), que se incorpora en 1994 coincidiendo con la puesta en marcha del LEADER 2 y que también se ha mantenido dentro de las siguientes convocatorias de la iniciativa comunitaria (ver datos sobre financiación por medidas en ambos territorios para las convocatorias LEADER 2 y LEADER plus en el Anexo).

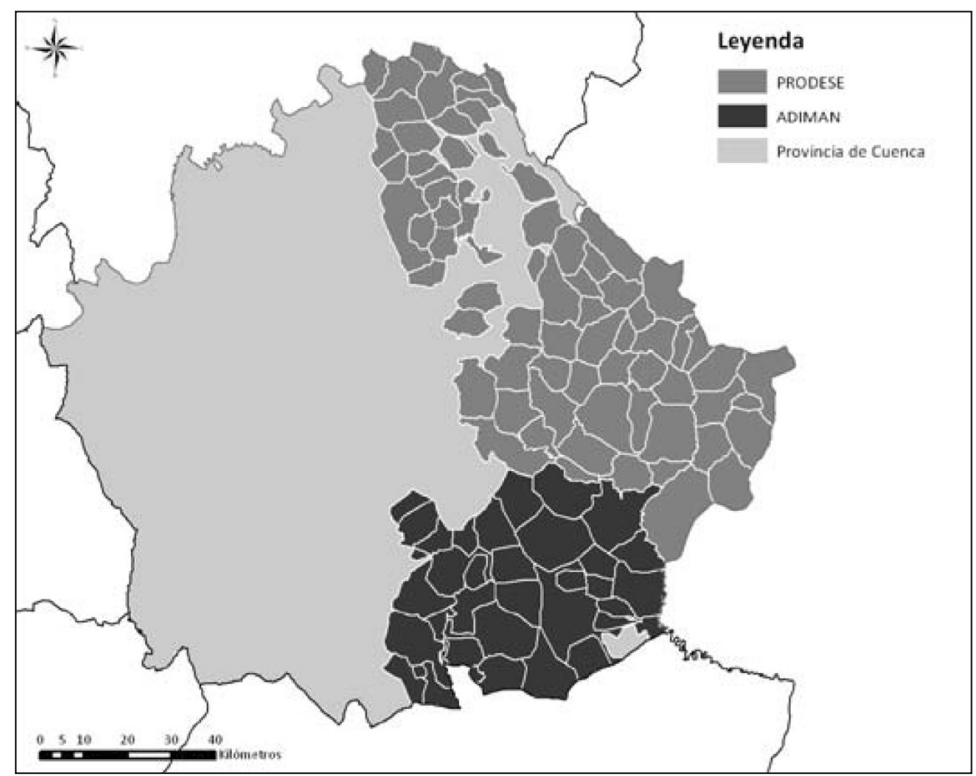

Mapa 1. Situación y estructura municipal de los territorios analizados.

Fuente: elaboración propia. 
A partir de los datos oficiales del padrón del año 2007, se identificó una muestra a escala municipal de 242 personas que respondieron a cuestionarios telefónicos (151 en el territorio de la Manchuela conquense y 91 en la Serranía de Cuenca), completada con 20 entrevistas semi-estructuradas con informantes clave que nos han servido realizar cruces de verificación con el fin de garantizar la cohesión territorial de los resultados. Básicamente, en nuestro proyecto hemos pretendido identificar los niveles de capital social a partir de los datos obtenidos sobre confianza, normas compartidas, características de las redes sociales y niveles de civismo. Tras una primera identificación hemos tratado de establecer correlaciones con el grado de desarrollo de cada comarca a escalas micro (municipio) y meso (comarca), teniendo en cuenta otras dimensiones del "capital humano" (distribución de la población, niveles de formación, estructura socioeconómica y del mercado de trabajo, población inmigrante), del "capital cultural" (patrimonio cultural tal como es percibido y valorado por la población local), y "capital físico" (límites y oportunidades, desarrollo de las infraestructuras, disponibilidad y accesibilidad de los servicios, y calidad/ conservación del medio natural).

En síntesis las distintas fases del proceso investigador han sido las siguientes:

a. Análisis demográfico y socio-económico de las fuentes de datos cuantitativos manejados en la primera fase de la investigación.

b. Análisis y clasificación de las actividades económicas.

c. Estudios y tratamiento de las redes asociativas y de cooperación a partir de la depuración del Directorio de Asociaciones para cada una de las comarcas analizadas.

d. Cuestionario telefónico sobre capital social a una muestra representativa de residentes ( 242 personas) proporcionalmente distribuida en cada uno de los municipios que integran las distintas comarcas de la provincia. Realizada entre mayo y octubre de 2008.

e. Entrevistas semi-estructuradas en profundidad a una "muestra estratégica positiva" y a una muestra de empresarios locales. La muestra estratégica incluye aquellos actores que conocen el territorio y pueden proporcionar información útil sobre los procesos sociales así como sobre los procesos relacionados con los sistemas socio-productivos en cada una de las comarcas.

f. Focus group con expertos y actores locales estratégicos.

Obviamente, al objeto de análisis que abordamos en este comunicación, sólo hemos recurrido a parte de los materiales y datos generados en el proceso investigador, centrándonos de manera específica en los resultados del cuestionario telefónico sobre participación, comunidad e identidad, así como en los datos recogidos sobre la distribución de los fondos LEADER para cada territorio, sin olvidar las matizaciones y aportaciones realizadas por los entrevistados dentro de la muestra estratégica de la cada uno de los dos territorios. 


\section{Presentación de los territorios de estudio}

El territorio vinculado al grupo de acción local PRODESE presenta, desde el punto de vista del espacio físico y del paisaje dos comarcas de rasgos diferenciados, la Serranía Alta y la Serranía Baja, ambas enmarcadas en la rama castellana del Sistema Ibérico, sin embargo, desde el punto de vista económico, social, cultural e histórico ambas presentan elementos comunes, por lo que es posible afirmar que pese a su gran extensión y diferenciación paisajística y agraria la zona de actuación mantiene una idiosincrasia que le es característica y definitoria. Dentro de este extenso territorio que se configura hoy como un desierto demográfico la sangría poblacional se ha mantenido incontenible, incluso tras la aplicación de las iniciativas LEADER (ver cuadros 1 y 2), aunque la situación es desigual a escala local. Casi un 50\% de los 72 municipios que lo integran presentan una densidad inferior a los $3,6 \mathrm{hab} / \mathrm{km}^{2}$ y son bastantes más los situados por debajo de la media. En términos de dimensión municipal, la media es de 240 habitantes/municipio siendo mayoría amplia aquellos que no llegan al 60\% de esa dimensión media, concretamente 27 municipios tienen una población inferior a los 100 habitantes. Las distancias intermunicipales existentes, en un marco de orografía de montaña, hacen que se hable de un hábitat rural disperso con tendencia al aislamiento intra-comarcal. Esta característica es una clara debilidad a la hora de plantear cualquier política municipal. De hecho, expertos en desarrollo rural opinan que por debajo de los $9 \mathrm{hab} / \mathrm{km}^{2}$ un municipio no es sostenible puesto que no puede garantizar el funcionamiento de servicios cotidianos para sus habitantes. Así, la situación es todavía más sangrante si nos atenemos a las palabras de los técnicos del GAL "el Padrón municipal es demasiado optimista en relación a la población de los municipios, ya que la que reside realmente en éstos es mucho menor, aproximadamente el $50 \%$ de la población no reside en el municipio".

Cuadro 1. Evolución de la población entre 1950 y 2012

\begin{tabular}{|l|r|r|r|r|r|}
\hline $\begin{array}{c}\text { Grupos de Desarrollo } \\
\text { Rural }\end{array}$ & $\begin{array}{c}\text { Censo } \\
\mathbf{1 9 5 0}\end{array}$ & $\begin{array}{c}\text { Censo } \\
\mathbf{1 9 9 1}\end{array}$ & $\begin{array}{c}\text { Padrón } \\
\mathbf{2 0 1 2}\end{array}$ & $\begin{array}{c}\text { Evolución } \\
\text { 1950-1991 } \\
(1950=100)\end{array}$ & $\begin{array}{c}\text { Evolución } \\
\text { 1991-2012 } \\
(1991=100)\end{array}$ \\
\hline ADIMAN & 61.889 & 37.678 & 42.715 & 60,9 & 113,4 \\
\hline PRODESE & 53.776 & 20.412 & 17.232 & 38,0 & 84,4 \\
\hline Provincia de Cuenca & 344.033 & 201.095 & 218.036 & 58,4 & 108,4 \\
\hline
\end{tabular}

Fuente: INE. 
Cuadro 2. Datos territoriales básicos

\begin{tabular}{|l|r|r|r|}
\hline \multicolumn{1}{|c|}{$\begin{array}{c}\text { Grupos de } \\
\text { Desarrollo Rural }\end{array}$} & \multicolumn{3}{|c|}{ Datos Territoriales (2012) } \\
\hline & $\begin{array}{c}\text { Número de } \\
\text { municipios }\end{array}$ & Superficie $\left(\mathrm{km}^{2}\right)$ & $\begin{array}{c}\text { Densidad de } \\
\text { población (h/km²) }\end{array}$ \\
\hline ADIMAN & 33 & 2.653 & 16,4 \\
\hline PRODESE & 72 & 4.749 & 3,6 \\
\hline Provincia de Cuenca & 238 & 17.141 & 12,7 \\
\hline
\end{tabular}

Fuente: INE.

La situación de la comarca viene determinada por su contexto socioeconómico. La necesidad de buscar alternativas de ingresos en otros sectores diferentes al industrial, la disminución de la natalidad al existir escasa población en edad fértil, la apuesta por la valorización de los recursos naturales como fuente de ingreso... explican la decisión del grupo de acción local de concentrar esfuerzos dentro de los siguientes bloques temáticos: infraestructuras turísticas, concienciación ciudadana, modernización y embellecimiento de núcleos rurales, y formación.

Según los datos del Ministerio de Trabajo e Inmigración (Tesorería General de la Seguridad Social, diciembre de 2007), en la Serranía de Cuenca hay un total de 475 empresas. Dejando aparte el sector primario (en situación de retroceso tanto para la agricultura como para la ganadería) y el turístico, en la Serranía Conquense existe un tejido empresarial bastante diversificado, en el que destacan algunas industrias agroalimentarias. Sin embargo el conjunto de actividades empresariales aparece totalmente fragmentado: a) faltan industrias de transformación que aprovechen la producción del sector primario no agrario; b) el tamaño o dimensión media es muy bajo hablándose con más propiedad de microactividades; c) aparecen en bastantes lugares pero con amplios vacíos en el conjunto del territorio; d) ninguno de ellos ejerce tracción sobre otras actividades; y e) hay una fuerte duplicidad de oferta de actividades y de mercados de destino máxime si estos son locales. Las cooperativas juegan un papel fundamental en la economía agraria y en el mundo rural; a menudo son las únicas empresas ubicadas en zonas rurales, por lo que generan riqueza y fijan la población en el territorio. Pero además de esta fuerte connivencia económica, las cooperativas tienen importantes implicaciones sociales y medioambientales. Sin embargo, en la comarca sólo hay 9 cooperativas, dedicadas al sector del aceite, el cereal y el vino, sin que pueda hablarse de predominio de ninguno de estos sectores. No existe ninguna Sociedad Agraria de Transformación en la zona.

En la Serranía de Cuenca hay un total de 75 industrias agroalimentarias de las cuales la gran mayoría se localizan en Landete y Talayuelas. En cuanto a 
su tipología destacan fundamentalmente aquellas industrias relacionadas con la vocación ganadera del territorio: la industria de la alimentación animal, la cárnica y huevos con 27 y 9 instalaciones respectivamente. Todo ello, sin dejar a un lado el sector de la panadería, el principal en todo el territorio castellanomanchego por abastecer a la población de un bien de primera necesidad. No obstante, y a pesar de los marchamos de calidad que ofrecen estos productos, la gran asignatura pendiente es la promoción, comercialización e internacionalización de los mismos.

Respecto al tejido y redes asociativas, la mayor parte de personas que deciden pertenecer y actuar dentro de algún tipo de colectivo lo hacen en las asociaciones de padres (en relación con el sistema educativo formativo) y sociales (jubilados, amas de casa y culturales). Entre los jóvenes apenas existe actividad asociativa permanente, especialmente entre las de tipo cultural, ya que la mayoría de éstas suelen crearse para actuaciones puntuales como la organización de las fiestas populares, o las que se crean con el objetivo de solicitar una determinada subvención.

Por otra parte, el patrimonio etnográfico de toda la comarca se caracteriza por su gran homogeneidad, además, a diferencia de otras comarcas la existencia hasta hace unos 150 años de una unidad administrativa propia ha permitido fortalecer ese sentimiento de pertenencia a una cultura determinada y hacer de la memoria histórica un motor de dinamización social. Así pues, tanto el Patrimonio Cultural material como el etnográfico constituyen un importante capital del territorio que se encuentra indebidamente valorado y que puede ser una oportunidad de desarrollo de la calidad de vida para los habitantes de la Serranía. Otra categoría de patrimonio estrechamente ligada a esta zona es el paleontológico, ya que la comarca presenta un amplio número de yacimientos entre los que destacan Lo Huеco en Fuentes, considerado el yacimiento de dinosaurios más importante de la Península Ibérica, con más de 6.000 piezas de diversas especies aparecidas hasta la fecha, entre ellas cocodrilos o tortugas, así como el yacimiento de Portilla y el de Las Hoyas, en el municipio de La Cierva, contado todos ellos con un gran potencial turístico. También cabe destacar en este apartado el arte rupestre y patrimonio geológico de Villar del Humo (las pinturas rupestres fueron declaradas Patrimonio de la Humanidad en 1998 dentro del conjunto de Arte Rupestre Levantino), uno de los elementos singulares del territorio con posibilidad de constituirse como uno de los recursos vertebradores del mismo. Sin embargo, al igual que ocurría con el sector agroalimentario, se observa un problema de comercialización en el sector del turismo que carece de la venta conjunta de paquetes turísticos, que incluyan alojamiento, restauración y actividades complementarias.

Desde un punto de vista teórico, la figura de protección se entiende en las estrategias de desarrollo del capital natural del territorio como un paso previo para articular a partir de ahí un desarrollo de las potencialidades del mismo. 
La declaración de zona protegida con este enfoque, es un pretexto para abrir un proceso de comunicación y de construcción colectiva de decisiones sobre qué hacer con el capital natural que existirá a futuro, y "cómo se puede poner a trabajar a ese capital natural a favor de la economía y el bienestar sociales del territorio". Estas figuras de protección son el inicio de la introducción de otras figuras y estrategias de corte transversal (naturaleza y turismo, espacios potenciales de producción de elementos de gran valor, etc.), y sobre todo para la Ordenación del Territorio hacia esa imagen y atractivo que se ha citado anteriormente. En el caso de la Serranía de Cuenca el capital natural es algo reconocido y valorado desde comienzos del siglo XX, cuando afamados naturalistas y geógrafos comenzaron el estudio y valoración de las cuencas del Júcar y el Cabriel y la parte superior del Tajo. Fruto de ello fue una concienciación de las administraciones, que a lo largo de los últimos treinta años ha llevado a que éstas incluyan en las diferentes figuras de protección del capital natural los abundantes recursos de la Serranía. En este sentido, en la actualidad casi el 56\% de la superficie de la comarca y más del 74\% de lo que sería la Serranía propiamente dicha aparece como superficie protegida. Entre los espacios naturales protegidos destaca por su proyección el Parque Natural de la Serranía de Cuenca (con una superficie de 73.726 ha agrupa 10 municipios de la Serranía y parte del término municipal de la propia capital provincial), al que habría que añadir una Reserva Natural, siete Monumentos Naturales y cuatro Microrreservas. En síntesis, las malas comunicaciones y el aislamiento del territorio durante tanto tiempo, ha contribuido a que en la actualidad el entorno natural y patrimonial se encuentre relativamente bien conservado, y sea uno de los principales recursos y potenciales con los que cuenta la Comarca. No obstante, y a pesar de la calidad de todos los recursos patrimoniales apuntados, tanto culturales como ambientales, su aprovechamiento turístico actual es escaso.

El segundo de los dos territorios analizados, la Manchuela conquense, gestionada por el grupo de acción local ADIMAN, es parte de una comarca natural bien definida pero cuya situación político-administrativa ha producido históricamente una fractura en su identidad. La pertenencia de La Manchuela a dos provincias (Cuenca y Albacete) propicia el que algunos municipios no se decanten por una de ellas (El Herrumblar), la falta de límites naturales sobre todo en el sur de la comarca contribuye también a que la interiorización del concepto zonificación y el trabajo municipal en torno a esta unidad no sea algo asentado y asumido por la población, que ve sobre todo la escala local como principal horizonte territorial. La inclusión en la Manchuela conquense por su flanco norte de municipios más acordes con la Serranía baja tampoco ayuda a la cohesión conceptual y explica que los diferentes mapas de zonificación de servicios presenten una gran heterogeneidad.

En esta unidad comarcal residían a principios de 2012 un total 42.715 personas, lo que lleva a una densidad de $16,4 \mathrm{hab} / \mathrm{km}^{2}$. Esta densidad se aproxima 
ya a lo que se considera como hábitat semiurbano (cuadros 1 y 2) y se sitúa por encima de la media de Cuenca $\left(12,7 \mathrm{hab} / \mathrm{km}^{2}\right)$, pero lejos de la media española. Los municipios de la comarca son municipios de dimensión pequeña: hay 11 con población mayor de 1.000 habitantes -menos de un tercio del total de municipios de la Manchuela conquense- y entre ellos sólo aparecen tres con más de 4.000 habitantes (2 si sólo se consideran los $>5.000$, Motilla del Palancar y Quintanar del Rey), si bien es cierto que estos últimos han experimentado un crecimiento sostenido tanto en el período 1950-1991 como desde esta última fecha hasta el año 2012, etapa en la que el crecimiento se ha difundido a buena parte de los municipios mayores de 1.000 habitantes y que explica que, en conjunto, la evolución de la comarca haya sido positiva, con Motilla del Palancar ejerciendo de cabecera comercial y de servicios. El origen y las causas de este crecimiento son debidas, por un lado, al aumento de población producido en determinados municipios de la comarca que han visto favorecido su desarrollo a partir de diferentes actividades económicas (servicios, construcción, algo de industria...), y, por otro lado, al aporte de población inmigrante $(15,6 \%$ de inmigrantes extranjeros sobre la población total de la comarca en 2011), factor que puede ser considerado como un estímulo para ayudar a fijar la población en las zonas rurales de este territorio. De entre los municipios que más han aumentado la población en este período se encuentra Quintanar del Rey, Pozoamargo, Graja de Iniesta, Iniesta y Motilla del Palancar. Las causas de este aumento de población en cada uno de los municipios son distintas atendiendo a las diferentes situaciones. Por ejemplo, en Motilla del Palancar en los últimos 15 años ha habido un incremento de actividad económica de cara a mejorar los servicios prestados a la población del municipio, y así a los municipios aledaños. Esta demanda de servicios, ha generado un aumento de actividades económicas, suponiendo un aumento de la oferta de puestos de trabajo y un flujo migratorio de personas desde municipios aledaños. En el caso de Quintanar del Rey, el municipio más poblado de la comarca, al sector de la construcción se ha erigido en el motor básico de desarrollo de los últimos años. Los puestos de trabajo generados en este ámbito, junto al de la agricultura y el champiñón, han dado como resultado una fijación de la población en el municipio que genera una serie de demandas en cuanto a servicios, que a su vez han derivado en actividades económicas y puestos de trabajo.

En el conjunto de la Manchuela conquense, el empleo se da principalmente en los servicios asociados a la atención a la población y a la agricultura. Entre los datos manejados (ver cuadro 3) destaca el fuerte empleo en la construcción. Habida cuenta de que la construcción en infraestructuras y edificaciones en la comarca no tiene la capacidad de absorber tal cantidad de empleo, hay que pensar que gran parte de él se localiza o más bien localizaba fuera de la misma implicando una fuerte demanda de movilidad entre el lugar de residencia y el de trabajo (obra). En la actualidad, una vez superado el "boom" de la construc- 
ción de edificios y viviendas, no parece haber actividad suficiente como para mantener el empleo en la construcción en valores superiores al de la industria. Por tanto, la situación del empleo a futuro es de alta incertidumbre y de riesgo de aumento del paro sectorial (la tasa de paro en la comarca pasó de un 7,8\% en 2006 a un 13,2\% en marzo de 2011).

Cuadro 3. Estructura del mercado de trabajo

\begin{tabular}{|c|r|r|r|r|r|r|r|r|}
\hline $\begin{array}{c}\text { Grupos de } \\
\text { Desarrollo } \\
\text { Rural }\end{array}$ & \multicolumn{7}{|c|}{ Estructura del Mercado de trabajo (diciembre 2007) } \\
\hline & $\begin{array}{c}\text { Trabajadores } \\
\text { en el sector } \\
\text { primario }\end{array}$ & $\%$ & $\begin{array}{c}\text { Trabajadores } \\
\text { en la } \\
\text { industria }\end{array}$ & $\%$ & $\begin{array}{c}\text { Trabajadores } \\
\text { en la } \\
\text { construcción }\end{array}$ & $\%$ & $\begin{array}{c}\text { Trabajadores } \\
\text { en el sector } \\
\text { terciario }\end{array}$ & $\%$ \\
\hline ADIMAN & 3.668 & 24,2 & 1.843 & 12,2 & 3.295 & 21,8 & 6.342 & 41,9 \\
\hline PRODESE & 873 & 20,7 & 919 & 24,3 & 513 & 16,4 & 1.312 & 38,6 \\
\hline
\end{tabular}

Fuente: Ministerio de Trabajo e Inmigración 2007.

La falta de mentalidad innovadora y espíritu emprendedor son otros de los rasgos que caracterizan a la población agrícola de la comarca así como al resto de la región de Castilla La Mancha. Los proyectos innovadores, planteados como alternativa y complemento al sistema agrícola actual, se ven frenados en cierta medida por falta de apoyo del sector agrícola (agricultores y cooperativas) y, sin embargo, es necesario abordar una industrialización de las cooperativas agrícolas de la comarca para que sus productos sean competitivos en un mercado global. Paradójicamente, el elevado número de cooperativas existentes (21 cooperativas, 10 bodegas y 2 almazaras) es un claro ejemplo del problema de atomización. Esta gran división frena el desarrollo de productos como el vino, que, siendo el gran potencial de la región, posee un grave problema de comercialización del producto derivado de la oferta y demanda del sector.

La Manchuela conquense, con 3 plantas de producción de setas Pleurotus ostreatus, es la comarca que más setas produce de Europa, con aproximadamente $12.000 \mathrm{Tm}$ de producción. Destacar además que en la Manchuela conquense se encuentra el primer centro de investigación de champiñón de España, conseguido mediante un acuerdo entre Diputación, Consejería de Agricultura y el sector del champiñón, ubicado en Quintanar del Rey, al que se suman 52 empresas dedicadas al sector. Empero, si se analiza la evolución del sector desde sus inicios, se constata que Manchuela conquense no ha sabido mantener su liderazgo de posicionamiento. La falta de una estrategia de comercialización y puesta en el mercado del producto ha hecho que hoy día, sus precios dejen un margen bastante escueto para el productor. 
Esta atomización general de la estructura empresarial se refleja en el hecho de que más del 50\% de la población ocupada de la Manchuela conquense son empresarios. De esto se deduce que la población de la comarca está acostumbrada a llevar a cabo su propio negocio: agricultores, carpinteros, herreros, mecánicos, panaderos... En los pueblos de la Manchuela conquense se dan una serie de actividades económicas, cuyo foco original son la agricultura y la ganadería, y a partir del cual, se generan una serie de servicios asociados para la población. Los índices industriales en la comarca son bajos, y estas actividades se concentran en los pueblos más grandes. No ocurre lo mismo con la construcción, actividad económica importante y actualmente generadora de empleo en la comarca.

El problema de vertebración antes mencionado también se refleja en las redes formales existentes entre la población, de la lista de asociaciones manejada, aproximadamente el $20 \%$ se encuentran funcionando de forma activa y a lo largo de todo el año. El resto se trata de asociaciones que se forman para acontecimientos puntuales (fiestas locales, procesiones...), de escala mayoritariamente local, y en las que el tema del trabajo de la participación con los socios sigue siendo una asignatura pendiente.

Para acabar de caracterizar la comarca, cabe señalar que los recursos culturales de la Manchuela conquense constituyen una valiosa fuente de su pasado. Son el recuerdo histórico de todo lo acontecido en la comarca, el reflejo de su sentir artístico, religioso y lleva encadenado el trabajo y dedicación de muchos de sus habitantes, por lo que constituyen un factor clave en términos identitarios. Dentro de la comarca existen enclaves o términos municipales principales en cuanto a la existencia de recursos culturales como son: Alarcón, Villanueva de la Jara e Iniesta, donde se apuesta por un adecuado estado de conservación de los monumentos históricos y una posterior puesta en valor. En conjunto todos ellos serían susceptibles de activarse y articularse para crear productos turísticos ofreciendo a la comarca una alternativa de desarrollo sostenible y complementario, sin embargo, hasta la fecha, debido al potencial de recursos naturales y patrimoniales con los que contaban, sólo Alarcón y Enguídanos, han creado una red de infraestructuras capaz de ofrecer una mínima oferta atractiva para el turista.

\section{COMUNIDAD, PARTICIPACIÓN E IDENTIDAD: LAS DIMENSIONES RELACIONALES, COGNI- TIVAS Y ESTRUCTURALES DEL CAPITAL SOCIAL}

Partiendo de que la confianza, reciprocidad y solidaridad son elementos clave de las dimensiones relacionales del capital social, hemos tratado de medirlas para valorar su papel en apoyo de los mecanismos de intercambio y cooperación dentro de la comunidad. Entre nuestros primeros hallazgos se encuentra el hecho de que, aparentemente, la confianza y la reciprocidad/solidaridad no están necesariamente correlacionadas. En el caso de Prodese, presenta altos 
niveles de confianza en la administración provincial y muy bajos en el Grupo de Acción Local, a pesar de haber estado presente en el territorio gestionando la iniciativa comunitaria Leader desde 1991, sin embargo, cuando abordamos la confianza en el futuro de la comarca, la Manchuela conquense, el territorio más dinámico de la provincia, alcanza las puntuaciones más altas, que contrastan con su desconfianza hacia las instituciones regionales, provinciales y europeas.

La reciprocidad y la solidaridad parecen más presentes en la comunidad de la Manchuela, especialmente cuando se trata de contribuir con tiempo al desarrollo de proyectos locales (cuadro 4). Por el contrario, los entrevistados en el territorio de Prodese parecen mucho más reticentes a participar en actividades

Cuadro 4. Reciprocidad y solidaridad

\begin{tabular}{|l|c|c|}
\hline & ADIMAN & PRODESE \\
\hline $\begin{array}{l}\text { ¿Ha participado usted u otro miembro de su familia en } \\
\text { actividades en las que la gente trabaja unida en beneficio } \\
\text { de la comunidad local? }\end{array}$ & Yes/No & Yes/No \\
\hline
\end{tabular}

Categorías de respuesta: $1=$ Sí, y $2=$ No.

\begin{tabular}{|l|c|c|c|c|}
\hline & \multicolumn{2}{|c|}{ ADIMAN } & \multicolumn{2}{c|}{ PRODESE } \\
\hline & Media & Desviación Estadística & Media & Desviación Estadística \\
\hline ¿con qué frecuencia? & 2,16 & 0,93 & 1,57 & 0,53 \\
\hline
\end{tabular}

Categorías de respuesta: $1=$ una vez al año; $2=$ varias veces al año; $3=$ mensualmente; $4=$ semanalmente, y $5=$ diariamente.

\begin{tabular}{|c|c|c|c|c|}
\hline & \multicolumn{2}{|c|}{ ADIMAN } & \multicolumn{2}{c|}{ PRODESE } \\
\hline & Media & $\begin{array}{c}\text { Desviación } \\
\text { Estadística }\end{array}$ & Media & $\begin{array}{c}\text { Desviación } \\
\text { Estadística }\end{array}$ \\
\hline $\begin{array}{l}\text { ¿Cómo valora la actitud de los vecinos } \\
\text { a la hora de trabajar juntos y cooperar? }\end{array}$ & 2.79 & 0.65 & 2.71 & 0.50 \\
\hline
\end{tabular}

Categorías de respuesta: $1=$ Muy desfavorable, $2=$ desfavorable, $3=$ favorable; y $4=$ muy favorable.

\begin{tabular}{|l|c|c|}
\hline $\begin{array}{c}\text { En el caso de que un proyecto local no le beneficiara } \\
\text { directamente pero resultara beneficioso para mucha gente } \\
\text { del pueblo ¿estaría dispuesto a contribuir con...? }\end{array}$ & ADIMAN & PRODESE \\
\hline & Sí/No & Sí/No \\
\hline Tiempo & 1,22 & 1,33 \\
\hline Dinero & 1,74 & 1,66 \\
\hline
\end{tabular}

Categorías de respuesta: $1=$ Sí, y $2=$ No 
en las que las personas tienen que trabajar juntas en beneficio de la comunidad. Del análisis de los cuestionarios parece desprenderse que los conceptos de reciprocidad y solidaridad tiene una faceta pragmática de interés común más tangible e instrumentalizable que el concepto genérico de confianza, que en algunos casos se da por hecha, de no mediar experiencias previas negativas. Todo ello parece consistente con la teoría del capital social, que considera que el índice de confianza presenta una correlación inversa al tamaño del grupo y su cohesión, no por casualidad el tamaño medio de los municipios de Prodese (242 habitantes) es mucho menor que los de Adiman (1.294 habitantes). Contradicciones similares aparecen en algunas teorías sobre el papel del capital social en áreas de montaña, distintos especialistas subrayan que las regiones montañosas tienen, en términos comparativos con otros espacios rurales, sistemas de relaciones comunitarias fuertes (MAGNANI y STRUFFI, 2009), mientras otros afirman que las comunidades residentes en estos espacios son a menudo "sociedades divididas" (OSTI, 2000), donde el cambio acelerado por la modernización ha exacerbado antiguas rivalidades familiares con nuevos conflictos entre categorías profesionales (agricultores, ganaderos, empresarios turísticos) que persiguen distintos y a menudo contradictorios objetivos económicos.

El componente cognitivo del capital social parece jugar un papel crucial a la hora de reducir los riesgos relacionados con la puesta en marcha de un proyecto común. Si examinamos los resultados del cuestionario relativos a normas y valores compartidos se repite el patrón antes analizado para el caso de la confianza (cuadro 5), los valores más altos se concentran en la Serranía donde

Cuadro 5. Valores, normas compartidas, y confianza generalizada

\begin{tabular}{|l|c|c|c|c|}
\hline $\begin{array}{c}\text { En general, iestá usted de acuerdo con las } \\
\text { siguientes afirmaciones? }\end{array}$ & \multicolumn{2}{|c|}{ ADIMAN } & \multicolumn{2}{c|}{ PRODESE } \\
\hline & Media & $\begin{array}{c}\text { Desviación } \\
\text { Estadística }\end{array}$ & Media & $\begin{array}{c}\text { Desviación } \\
\text { Estadística }\end{array}$ \\
\hline $\begin{array}{l}\text { La mayor parte de los vecinos del pueblo } \\
\text { están dispuestos a ayudar cuando se les } \\
\text { necesita }\end{array}$ & 2,86 & 0,37 & 2,79 & 0,44 \\
\hline $\begin{array}{l}\text { En este pueblo tiene que estar alerta o } \\
\text { cualquiera puede aprovecharse de usted }\end{array}$ & 2,49 & 0,52 & 2,11 & 0,32 \\
\hline $\begin{array}{l}\text { En general, la gente se comporta siguien- } \\
\text { do normas adecuadas y compartidas de } \\
\text { civismo }\end{array}$ & 2,87 & 0,33 & 2,93 & 0,29 \\
\hline Puede fiarse de la mayor parte de la gente & 2,78 & 0,45 & 2,80 & 0,40 \\
\hline
\end{tabular}

Categorías de respuesta: $1=$ muy en desacuerdo; $2=$ en desacuerdo; $3=$ de acuerdo; $y$ 4= totalmente de acuerdo. 
la mayor parte de la gente parece dispuesta a ayudar cuando se la necesita o comportarse siguiendo normas adecuadas de civismo, y por descontado afirman que abrirían sus puertas a cualquiera que quisiera instalarse en el pueblo. No podemos olvidar que en este territorio el peso de la inmigración es extremadamente bajo, así como el hecho de que en este tipo de comunidades el "control social" sobre los comportamientos esperados es muy fuerte.

Un patrón opuesto y muy significativo aparece al analizar las respuestas sobre el grado de identificación territorial/institucional (cuadro 6). Aparentemente el mayor grado de identificación territorial se da entre los residentes de la Manchuela, que aseguran estar bastante o muy satisfechos con su pueblo (micro-escala) así como con la región y país (macro-escala). ¿Podríamos deducir de estos datos una relación más equilibrada entre vinculación o pertenencia a grupos similares (bonding) y la conexión con grupos o personas diferentes (bridging) en el caso de Adiman? (STORPER, 2005). Lamentablemente los resultados todavía no son lo suficientemente sólidos en esta fase de la investigación, que deberemos profundizar en el futuro. Por otro lado, son sorprendentes los pobres resultados de Prodese en relación con su identidad social y territorial, especialmente si tenemos en cuenta su fuerte personalidad desde el punto de vista físico e histórico y el hecho de haber estado recibiendo fondos de la iniciativa LEADER desde 1991 (ver anexo). Ciertamente la situación de aislamiento, despoblación y envejecimiento añadidas a la falta de iniciativas innovadoras para las explotaciones tradicionales parecen haber generado un notable desapego entre la mayoría de la población.

Cuadro 6. Identidad

\begin{tabular}{|l|c|c|c|c|}
\hline \multirow{2}{*}{$\begin{array}{c}\text { Puntúe del 1 al 4 hasta qué punto piensa } \\
\text { usted que la gente se siente identificada } \\
\text { con los siguientes territorios/instituciones }\end{array}$} & \multicolumn{2}{|c|}{ ADIMAN } & \multicolumn{2}{|c|}{ PRODESE } \\
\cline { 3 - 5 } & & $\begin{array}{c}\text { Desviación } \\
\text { Estadística }\end{array}$ & Media & $\begin{array}{c}\text { Desviación } \\
\text { Estadística }\end{array}$ \\
\hline Sus raíces familiares & 3,33 & 0,53 & 2,91 & 0,32 \\
\hline Su pueblo & 3,19 & 0,42 & 2,92 & 0,27 \\
\hline Su comarca & 3,00 & 0,37 & 2,87 & 0,37 \\
\hline Su sector profesional & 2,84 & 0,49 & 2,78 & 0,51 \\
\hline Su provincia & 2,84 & 0,59 & 2,86 & 0,34 \\
\hline Su comunidad autónoma & 3,04 & 0,38 & 2,83 & 0,44 \\
\hline España & 3,06 & 0,29 & 2,95 & 0,31 \\
\hline La asociación a la que pertenecen & 3,03 & 0.25 & 2,44 & 0,57 \\
\hline $\begin{array}{l}\text { En general, ise considera usted satis- } \\
\text { fecho con su pueblo? }\end{array}$ & 3,30 & 0,65 & 2,97 & 0,35 \\
\hline
\end{tabular}

Categorías de respuesta: $1=$ muy poco, $2=$ poco, $3=$ bastante; $y 4=$ mucho. 
Si aceptamos las tesis de distintos especialistas, no hay duda de que la participación en actividades sociales o, en otras palabras, la pertenencia es central para el bienestar. Parece asumido que la posición por defecto es participar y que se produce un problema si los individuos o grupos de individuos no participan. Sin embargo, otros autores argumentan que mientras la participación es vista como un indicador de inclusión social y compromiso, no es necesariamente cierto que la no-participación pueda equipararse con la exclusión social (SHORTALL, 2008). En cualquier caso, la literatura no es concluyente acerca de la relación entre vida asociativa y desarrollo económico. Por una parte, existen muchos casos de sociedades con un débil asociacionismo que se ha desarrollado bien y, por otra, ciertas formas de asociacionismo pueden obstaculizar el desarrollo económico.

En primer lugar, debemos reconocer que el número de asociaciones en ambos territorios tiene una importancia relativa, lo que importa es la intensidad de las relaciones y actividades que mantienen los individuos. El número de grupos, redes o asociaciones a los que nuestros entrevistados o sus familiares dicen pertenecer se correlaciona con el dinamismo territorial, con las mayores puntuaciones en la Manchuela conquense (cuadro 7). Esto confirmaría la hipótesis de que los agentes "expertos" tienden a funcionar por afinidades selectivas, dentro de las comunidades económicas. Cuando estas afinidades no existen (es decir, las personas con conocimientos son extremadamente escasas

Cuadro 7. Relaciones sociales, redes, organizaciones y asociaciones

\begin{tabular}{|l|c|c|c|c|}
\hline & \multicolumn{2}{|c|}{ ADIMAN } & \multicolumn{2}{c|}{ PRODESE } \\
\hline & Media & $\begin{array}{c}\text { Desviación } \\
\text { Estadística }\end{array}$ & Media & $\begin{array}{c}\text { Desviación } \\
\text { Estadística }\end{array}$ \\
\hline $\begin{array}{l}\text { ¿A cuántos grupos, redes o } \\
\text { asociaciones pertenece usted o } \\
\text { cualquier miembro de su familia? }\end{array}$ & 0,75 & 1,21 & 0,38 & 0,76 \\
\hline
\end{tabular}

Categorías de respuesta: $0=$ ninguno; $1=1$ grupo; $2=2$ grupos; $3=3$ grupos; $4=4$ grupos; $5=$ grupos, y $6=6$ grupos.

\begin{tabular}{|l|c|c|c|c|}
\hline & \multicolumn{2}{|c|}{ ADIMAN } & \multicolumn{2}{c|}{ PRODESE } \\
\hline & Media & $\begin{array}{c}\text { Desviación } \\
\text { Estadística }\end{array}$ & Media & $\begin{array}{c}\text { Desviación } \\
\text { Estadística }\end{array}$ \\
\hline $\begin{array}{l}\text { ¿Cómo valora usted la difusión de la } \\
\text { información sobre los programas y } \\
\text { acciones de desarrollo de la región? }\end{array}$ & 2,55 & 0,62 & 2,35 & 0,52 \\
\hline
\end{tabular}

Categorías de respuesta: $1=$ muy escasa, $2=$ escasa, $3=$ buena; y $4=$ muy buena. 
por lo que no pueden coincidir con nadie, o son extremadamente desconfiadas), el conocimiento se desperdicia y tendrá escasos efectos positivos. No obstante, otro tipo de relaciones sociales, no organizadas formalmente dentro de redes, como comer o beber con otros en el mismo pueblo, son muy comunes en el caso de la Serranía, donde quizás su escasa y envejecida población no ve la necesidad de unirse en redes formales o asociaciones ya que no están motivados por el beneficio u organizados con fines específicos. No deberíamos olvidar que en ocasiones las perspectivas del capital social privilegian la idea de que todo aquello de relevancia en la vida social puede reducirse a la racionalidad o la economía, aspecto en el que otros autores no coinciden.

\section{A MODO DE CONCLUSIONES}

La discusión presentada en esta comunicación se centra en la medición e interpretación de algunos indicadores de capital social (reciprocidad, valores y normas compartidas, identidad y redes) para poblaciones rurales que viven en territorios deprimidos en los que se han implementado iniciativas comunitarias LEADER. En primer lugar, tendríamos que mencionar que la "confianza" sólo puede ser entendida en su contexto territorial y socio-cultural y que las expectativas mutuas son el "cemento social" que aglutina la mayor parte de las pequeñas comunidades rurales. A este respecto, y en relación con el desarrollo socioeconómico, la reciprocidad, solidaridad y "confianza territorial" o confianza en el futuro de la comarca parecen erigirse en indicadores más sensibles que la confianza generalizada, personal o institucional.

Asimismo, merece la pena recordar que en el proceso de transferencia de habilidades, conocimientos y valores el pasado (la historia) y el futuro de cualquier comunidad/territorio juegan un papel clave en la trasmisión de normas culturales y sociales. El resultado del aprendizaje es el beneficio mutuo. A través de las prácticas y el comportamiento de grupo la comunidad se beneficia estimulando a los individuos así como los intereses y significados sociales de verificar su identidad, contribuyendo al mismo tiempo al bienestar y/o sostenibilidad de la comunidad.

En cualquier caso, los resultados de la implementación de los programas LEADER ofrecen resultados muy diversos, las razones pueden ser el grado de eficiencia de los equipos técnicos de los grupos de acción local, el excesivo tamaño de alguno de los territorios seleccionados (como es el caso de la Serranía conquense), situados en regiones montañosas y desarticuladas, y especialmente la necesidad de converger en problemas comunes de construcción territorial a partir de un número de personas y/o entidades con identidades y alianzas alternativas en relación con el problema en cuestión; en este marco la red de alianzas y de consensos sobre los que se basan siempre es precaria y dependiente de las condiciones del contexto. 
BIBLIOGRAFÍA

ÁRNASON, A.; SHUCKSMITH, M. y VERGUNST, J. (ed.) (2009): Comparing Rural Development. Continuity and Change in the Countryside of Western Europe, Perspectives on rural policy and planning Series, United Kingdom, Ashgate, 212 p.

COHEN, A. (1985): Belonging: identity and social organisation in British rural culture, Manchester, Manchester University Press.

MAGNANI, N. y STRUFFI, L. (2009): "Translation sociology and social capital in rural development initiatives. A case study from the Italian Alps", Journal of Rural Studies, 25, p. 231-238.

OSTI, G. (2000): "LEADER and partnerships: the case of Italy", Sociologia Ruralis, 40 (2), p. 172-180.

RAY, C. (1999): "Endogenous development in the era of reflexive modernity", Journal of Rural Studies, 15 (3), p. 257-267.

SHUCKSMITH, M. (2000): "Endogenous Development, Social capital and Social inclusion: Perspectives from LEADER in the UK", Sociologia Ruralis, 40 (2), p. 209-217.

STORPER, M. (2005): "Society, community and economic development", Studies in comparative international development, 39 (4), p. 30-57. 
ANEXO

LEADER II (1995-2001). INVERSIÓN Y FINANCIACIÓN (en pesetas y \%)

\begin{tabular}{|c|c|c|c|c|c|c|c|c|}
\hline \multirow{2}{*}{$\begin{array}{c}\text { MEDI- } \\
\text { DAS }\end{array}$} & \multicolumn{2}{|c|}{ COSTE TOTAL } & \multicolumn{2}{|c|}{ GASTO PÚBLICO } & \multicolumn{2}{|c|}{ GASTO PRIVADO } & \multicolumn{2}{|c|}{ OTRAS AYUDAS } \\
\cline { 2 - 7 } & Pesetas & $\begin{array}{c}\% \text { sobre } \\
\text { total }\end{array}$ & Pesetas & $\begin{array}{c}\% \text { sobre } \\
\text { medida }\end{array}$ & Pesetas & $\begin{array}{c}\% \text { sobre } \\
\text { medida }\end{array}$ & Pesetas & $\begin{array}{c}\% \text { sobre } \\
\text { medida }\end{array}$ \\
\hline
\end{tabular}

B1- Apoyo técnico al desarrollo rural.

\begin{tabular}{|l|r|r|r|r|r|r|r|r|}
\hline PRODESE & 83.900 .714 & 4,59 & 75.749 .809 & 90,29 & 8.150 .905 & 9,71 & 0 & 0,00 \\
\hline ADIMAN & 106.626 .115 & 4,14 & 96.256 .170 & 90,27 & 10.369 .945 & 9,73 & 0 & 0,00 \\
\hline
\end{tabular}

B2- Formación profesional y ayudas

\begin{tabular}{|l|r|r|r|r|r|r|r|r|}
\hline PRODESE & 28.336 .745 & 1,55 & 22.352 .763 & 78,88 & 5.983 .982 & 21,12 & 0 & 0,00 \\
\hline ADIMAN & 27.165 .542 & 1,05 & 23.343 .113 & 85,93 & 3.822 .429 & 14,07 & 0 & 0,00 \\
\hline
\end{tabular}

B3- Turismo rural

\begin{tabular}{|l|r|r|r|r|r|r|r|r|}
\hline PRODESE & 584.358 .313 & 32,00 & 265.769 .438 & 45,48 & 298.097 .125 & 51,01 & 20.491 .750 & 3,51 \\
\hline ADIMAN & 274.124 .887 & 10,63 & 80.476 .245 & 29,36 & 193.648 .642 & 70,64 & 0 & 0,00 \\
\hline
\end{tabular}

B4- Pequeñas empresas, artesanía

\begin{tabular}{|l|r|r|r|r|r|r|r|r|}
\hline PRODESE & 614.285 .365 & 33,63 & 289.955 .244 & 47,20 & 305.657 .818 & 49,76 & 18.672 .303 & 3,04 \\
\hline ADIMAN & 1.228 .004 .796 & 47,64 & 282.272 .940 & 22,99 & 915.516 .122 & 74,55 & 30.215 .734 & 2,46 \\
\hline
\end{tabular}

B5- Valorización y comercialización

\begin{tabular}{|l|r|r|r|r|r|r|r|r|}
\hline PRODESE & 273.733 .535 & 14,99 & 112.754 .776 & 41,19 & 160.978 .759 & 58,81 & 0 & 0,00 \\
\hline ADIMAN & 648.913 .645 & 25,17 & 234.745 .349 & 36,18 & 414.168 .296 & 63,82 & 0 & 0,00 \\
\hline
\end{tabular}

B6- Conservación y mejora del medio

\begin{tabular}{|l|r|r|r|r|r|r|r|r|}
\hline PRODESE & 241.761 .604 & 13,24 & 219.381 .098 & 90,74 & 22.380 .506 & 9,26 & 0 & 0,00 \\
\hline ADIMAN & 293.092 .717 & 11,37 & 224.835 .029 & 76,71 & 68.257 .688 & 23,29 & 0 & 0,00 \\
\hline
\end{tabular}

\section{B- Programa de innovación rural}

\begin{tabular}{|l|l|r|r|r|r|r|r|r|}
\hline PRODESE & 1.826 .376 .276 & 100,00 & 985.963 .128 & 53,98 & 801.249 .095 & 43,87 & 39.164 .053 & 2,14 \\
\hline ADIMAN & 2.577 .927 .702 & 100,00 & 941.928 .846 & 36,54 & 1.605 .783 .122 & 62,29 & 30.215 .734 & 1,17 \\
\hline
\end{tabular}

C- Cooperación Internacional

\begin{tabular}{|l|r|r|r|r|r|r|r|r|}
\hline PRODESE & 0 & 0,00 & 0 & 0,00 & 0 & 0,00 & 0 & 0,00 \\
\hline ADIMAN & 0 & 0,00 & 0 & 0,00 & 0 & 0,00 & 0 & 0,00 \\
\hline
\end{tabular}

\section{TOTAL PROGRAMA REGIONAL}

\begin{tabular}{|l|r|r|r|r|r|r|r|r|}
\hline PRODESE & 1.826 .376 .276 & 100,00 & 985.963 .128 & 53,98 & 801.249 .095 & 43,87 & 39.164 .053 & 2,14 \\
\hline ADIMAN & 2.577 .927 .702 & 100,00 & 941.928 .846 & 36,54 & 1.605 .783 .122 & 62,29 & 30.215 .734 & 1,17 \\
\hline
\end{tabular}

Fuente: Delegación de Agricultura de Cuenca. Junta de Comunidades de Castilla-La Mancha. LEADER + (2002-2006). INVERSIÓN Y FINANCIACIÓN (en euros y \%) 


\begin{tabular}{|c|c|c|c|c|c|c|}
\hline \multirow{2}{*}{ MEDIDAS } & \multicolumn{2}{|c|}{ COSTE TOTAL } & \multicolumn{2}{c|}{ GASTO PÚBLICO } & \multicolumn{2}{c|}{ GASTO PRIVAD0 } \\
\cline { 2 - 6 } & Euros & $\begin{array}{c}\% \text { sobre } \\
\text { total }\end{array}$ & Euros & $\begin{array}{c}\% \text { sobre } \\
\text { medida }\end{array}$ & euros & $\begin{array}{c}\% \text { sobre } \\
\text { medida }\end{array}$ \\
\hline
\end{tabular}

1.03. Servicios a la población

\begin{tabular}{|l|r|r|r|r|r|r|}
\hline PRODESE & $1.238 .876,54$ & 7,37 & $734.371,97$ & 59,28 & $504.504,57$ & 40,72 \\
\hline ADIMAN & $1.217 .266,95$ & 8,03 & $744.409,02$ & 61,15 & $472.857,93$ & 38,85 \\
\hline
\end{tabular}

1.04. Patrimonio natural

\begin{tabular}{|l|r|r|r|r|r|r|}
\hline PRODESE & $937.341,49$ & 5,57 & $705.351,00$ & 75,25 & $231.990,49$ & 24,75 \\
\hline ADIMAN & $935.289,32$ & 6,17 & $594.260,64$ & 63,54 & $341.028,68$ & 36,46 \\
\hline
\end{tabular}

1.05. Valorización de productos locales agrarios

\begin{tabular}{|l|r|r|r|r|r|r|}
\hline PRODESE & $839.916,04$ & 4,99 & $235.815,46$ & 28,08 & $604.100,58$ & 71,92 \\
\hline ADIMAN & $2.480 .065,81$ & 16,35 & $742.099,02$ & 29,92 & $1.737 .966,79$ & 70,08 \\
\hline
\end{tabular}

1.06. PYMEs y servicios

\begin{tabular}{|l|r|r|r|r|r|r|}
\hline PRODESE & $3.859 .266,54$ & 22,95 & $1.039 .358,99$ & 26,93 & $2.819 .907,55$ & 73,07 \\
\hline ADIMAN & $4.759 .800,50$ & 31,39 & $1.239 .171,04$ & 26,03 & $3.520 .629,46$ & 73,97 \\
\hline
\end{tabular}

1.07. Valorización del patrimonio cultural y arquitectónico

\begin{tabular}{|l|r|r|r|r|r|r|}
\hline PRODESE & $1.000 .097,33$ & 5,95 & $828.254,60$ & 82,82 & $171.842,73$ & 17,18 \\
\hline ADIMAN & $1.213 .775,36$ & 8,00 & $825.565,13$ & 68,02 & $388.210,23$ & 31,98 \\
\hline
\end{tabular}

1.08. Turismo

\begin{tabular}{|l|r|r|r|r|r|r|}
\hline PRODESE & $6.897 .255,82$ & 41,01 & $2.075 .816,30$ & 30,10 & $4.821 .439,52$ & 69,90 \\
\hline ADIMAN & $2.697 .809,18$ & 17,79 & $947.102,37$ & 35,11 & $1.750 .706,81$ & 64,89 \\
\hline
\end{tabular}

1.10. Formación y empleo

\begin{tabular}{|l|r|r|r|r|r|r|}
\hline PRODESE & $261.274,18$ & 1,55 & $202.758,98$ & 77,60 & $58.515,20$ & 22,40 \\
\hline ADIMAN & $368.987,54$ & 2,43 & $330.348,64$ & 89,53 & $38.638,90$ & 10,47 \\
\hline
\end{tabular}

1. Estrategias de Desarrollo
\begin{tabular}{|l|r|r|r|r|r|r|}
\hline PRODESE & $16.540 .487,53$ & 98,34 & $7.325 .571,42$ & 44,29 & $9.214 .916,11$ & 55,71 \\
\hline ADIMAN & $14.903 .555,45$ & 98,28 & $6.646 .991,57$ & 44,60 & $8.256 .563,88$ & 55,40 \\
\hline
\end{tabular}

2. Cooperación

\begin{tabular}{|l|r|r|r|r|r|r|}
\hline PRODESE & $279.069,01$ & 1,66 & $279.068,99$ & 100,00 & 0 & 0,00 \\
\hline ADIMAN & $260.675,75$ & 1,72 & $255.455,89$ & 98,00 & 5.220 & 2,00 \\
\hline
\end{tabular}

TOTAL MEDIDAS 1 y 2

\begin{tabular}{|l|r|r|r|r|r|r|}
\hline PRODESE & $16.819 .556,54$ & 100,00 & $7.604 .640,41$ & 45,21 & $9.214 .916,13$ & 54,79 \\
\hline ADIMAN & $15.164 .231,20$ & 100,00 & $6.902 .447,46$ & 45,52 & $8.261 .783,74$ & 54,48 \\
\hline
\end{tabular}

Fuente: Delegación de Agricultura de Cuenca. Junta de Comunidades de Castilla-La Mancha. 